\title{
Recombinant porcine norovirus identified from piglet with diarrhea
}

\author{
Quan Shen ${ }^{1,3}$, Wen Zhang ${ }^{1}$, Shixing Yang ${ }^{1}$, Zhibiao Yang ${ }^{1}$, Yan Chen ${ }^{2}$, Li Cui ${ }^{2}$, Jianguo Zhu ${ }^{2}$ and Xiuguo Hua ${ }^{*}$
}

\begin{abstract}
Background: Noroviruses (NoVs) are members of the family Caliciviridae and are emerging enteric pathogens of humans and animals. Some porcine NoVs are genetically similar to human strains and are classified into Gll, like most epidemic human NoVs. So far, PoNoV have been exclusively detected in fecal samples of adult pig without clinical signs.

Results: Result showed that 2 of the 12 evaluated fecal samples were positive for PoNoVs, one of which was positive for PoNoV alone, and the other was coinfected with porcine circovirus and PoNoV. Phylogenetic and recombination analysis showed that the PoNoV positive alone strain was a recombinant new genotype strain. Experimental infection of miniature pigs with fecal suspensions confirmed that this strain can cause gastroenteritis in piglets.

Conclusion: This is the first report that recombinant new genotype PoNoV exised in pig herd of China, which cause diarrhea in pigs in nature condition. This find raised questions about the putative epidemiologic role of PoNoV.
\end{abstract}

Keywords: Porcine norovirus, Recombinant, New genotype, Gastroenteritis

\section{Background}

Noroviruses (NoVs) are members of the family Caliciviridae and are emerging enteric pathogens of humans and animals $[1,2]$. They are small, non-enveloped viruses of $27-38 \mathrm{~nm}$ in diameter and possess a single-stranded, positive-sense genomic RNA. NoVs genome is 7.3-7.7 kb in length and contains 3 open reading frames (ORFs) [3]. ORF1 encodes a polyprotein that is autocatalytically cleaved to produce several proteins, including RNA-dependent RNA-polymerase (RdRp) and other non-structural proteins [4]. ORF2 encodes the major capsid protein, and ORF3 encodes for a minor structural protein [5]. NoVs have been isolated from humans and several species of animals, including swine, canine, bovine, murine and lion [6-8]. Based on sequence analyses comprising conserved regions within the $\mathrm{RdRp}$ and the capsid genes, NoVs are divided into 5 genogroups (GI-GV) and further subdivided into 27 genotypes [9]. Human NoVs are located in GI, GII and GIV. GII contains 19 genotypes, and

\footnotetext{
* Correspondence: huaxiuguo@yahoo.com

${ }^{2}$ Key laboratory of Veterinary Biotechnology, School of Agriculture and Biology, Shanghai JiaoTong University, 800 Dongchuan Road, Shanghai, People's Republic of China

Full list of author information is available at the end of the article
}

porcine strains are found in GII-11, -18 and -19 [10]. Some porcine NoVs are genetically related to human strains and are classified into GII, which contains most epidemic strains of NoVs in human. Some potential NoVs recombinant strains have been identified [10,11], which raises public health concerns regarding their potential for zoonotic transmission. To date, PoNoV have been exclusively detected in fecal samples of adult pig without clinical signs.

\section{Methods \\ Specimens}

Twelve fecal samples from piglets with diarrhea and no accurate of etiology were collected from three commercial pig farms in a Shanghai suburb, from May to August, 2009. In order to avoid sample contamination, specimens were obtained directly from the pig anus and disposable materials were used during sampling. Stool samples were freshly collected and immediately converted to $10 \%(\mathrm{w} / \mathrm{v})$ suspensions in PBS (0.01 $\mathrm{M}$ phosphate, $\mathrm{pH}$ 7.2-7.4, $0.15 \mathrm{M} \mathrm{NaCl}$,) for RNA and DNA extraction.

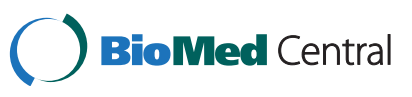




\section{RNA and DNA extraction}

RNA was extracted from $200 \mathrm{ul}$ of $10 \%$ fecal suspension by using the TRIzol reagent (Invitrogen, USA) following the manufacturer's instructions. RNA pellets were dissolved in 25ul RNase-free water and reverse transcription was performed immediately. DNA was extracted using The QIAamp DNA Stool Mini Kit (QIAGEN, Germany) according to the manufacturer's instructions.

\section{RT-PCR or PCR}

RT-PCR or PCR assays with different primer sets for the detection of PoNoV, and common viruses that can cause pig diarrhea including porcine circovirus type 2 , porcine rotavirus, porcine transmissible gastroenteritis virus, porcine sapovirus, and porcine epidemic diarrhea virus were performed as previously described [12-14]. The amplicons were analyzed by $1 \%$ agarose gel electrophoresis in TAE buffer, followed by staining with ethidium bromide $(0.5 \mu \mathrm{g} / \mathrm{ml})$ and visualization under UV light.

\section{Whole genome amplification}

The 3-kb $3^{\prime}$ end fragment of pNoVs-Ch6 was amplified with primers p290 and VN3T20 [10]. To amplify the remaining sequence, 6 sets of primers (Table 1) were designed based on the entire $\mathrm{PoNoV}$ genome sequence (AB126320) available in GenBank. Reverse transcription was carried out at $42^{\circ} \mathrm{C}$ for $1 \mathrm{~h}$ with $1 \mu \mathrm{l}$ (200 units) of AMV Reverse Transcriptase (TakaRa, Japan) and $1 \mu \mathrm{l}$ $(25 \mathrm{mM})$ of antisense primer. The PCR parameters of all amplification reactions included an initial incubation at $95^{\circ} \mathrm{C}$ for $5 \mathrm{~min}$, followed by 39 cycles of denaturation at $94^{\circ} \mathrm{C}$ for $1 \mathrm{~min}$, annealing for $1 \mathrm{~min}$ at a temperature varied according to the Tm of different primers, and extension at $72^{\circ} \mathrm{C}$ for $1.5 \mathrm{~min}$, with a final incubation at $72^{\circ} \mathrm{C}$ for $7 \mathrm{~min}$. PCR products were purified using the Axyprep DNA Gel Extraction Kit (AXYGEN, USA). The purified PCR products were ligated into PMD18-T vector (TakaRa, Japan) using T4 DNA ligase (TakaRa, Japan) at $16^{\circ} \mathrm{C}$ for $2 \mathrm{~h}$. The recombinant plasmid was transformed into DH5 $\alpha$ competent Escherichia coli cells (TakaRa, Japan). Three of the positive clones were sequenced.

\section{Sequence and recombination analysis}

Similarity searches of the sequences were carried out in BLAST (http://www.ncbi.nlm.nih.gov/BLAST/). After a multiple alignment with CLUSTAL W (version 1.4), the phylogenetic relationship of the strains in the present study and the reference isolates were assessed using the software MEGA Version 4.0. For analysis in MEGA, Jukescantor (JC) distance was utilized, employing the Neighbor joining (NJ) algorithm [15]. The reliability of different phylogenetic groupings was evaluated by using the bootstrap test (1000 bootstrap replications) available in MEGA. The identification of recombinants was
Table 1 Primers designed to amplify the remaining sequence of pNoVs-Ch6

\begin{tabular}{lll}
\hline Primer name & $\begin{array}{l}\text { Nucleotide } \\
\text { position }\end{array}$ & Nucleotide sequence $\mathbf{( 5}^{\prime} \mathbf{- 3}^{\prime} \mathbf{)}$ \\
\hline PNoV1F & $725-744$ & ACCTGTCCCTACAGTGGATG \\
PNoV1R & $1494-1513$ & ATATGAGTITGCCTATACC \\
PNoV2F & $1404-1425$ & ATCTCAGCAGCCAGGTCGCTCC \\
PNoV2R & $2154-2173$ & AACCCATACCTATTGTCAGC \\
PNoV3F & $2107-2126$ & TCTACAACTTCGATGTGGAC \\
PNoV3R & $2826-2845$ & TGACGGAGTCTCATCTCATC \\
PNoV4F & $2744-2762$ & CGAGGAGTACCTCCGTGAC \\
PNoV4R & $3552-3571$ & TGATTCTCACCACCCTCCAG \\
PNoV5F & $3525-3543$ & ACTCAGGGTCCTGATGGTG \\
PNoV5R & $4298-4318$ & TGCCTCTCTGTGGGTGGAG \\
PNoV6F & $4261-4279$ & ATACCTACCACTTTGATGC \\
PNoV6R & $4884-4902$ & ATGAGGCTTCTCCCAGCAGG \\
5'endF & $1-23$ & GTGAAATGAAGATGGCGTCTAAC \\
5'endR & $761-780$ & ATAGAATCACGTTGGCAACC \\
3'endF1(external & $7009-7031$ & ACTGGAATGGCACGAGATACTGG \\
forward primer) & & \\
5'endF2(internal & $7193-7212$ & ACTCTATGGGTACCTCTAG \\
forward primer) & & \\
5'endR & & TTITTIT \\
\hline Positon and & &
\end{tabular}

Position and nucleotide sequence of oligonucleotide primers for PCR. The nucleotide position is in accordance with $A B 126320$. In the primer names, $F$ and $\mathrm{R}$ mean sense primer and anti-sense primer, respectively.

performed by using the Recombination Detection Program (http://darwin.uvigo.es/rdp/rdp.html) [16]. Prototype NoVs strains used as references in the analysis and their GenBank accession numbers and source of origin are marked in the phylogenetic tree (Figure 1).

\section{Animals}

Eight 15-day old specific-pathogen-free (SPF) miniature pigs were purchased from Experimental Animal Center, School of Agricultural and Biology, Shanghai Jiaotong University (Shanghai, China), and maintained in a pathogen-free animal facility. This animal experiment was approved by the IACUC with a permit number of IACUC-2009-S-0202, which complies with the National Institute of Health Guide for the Care and Use of Laboratory Animals.

\section{Experimental infection}

The pNoVs-Ch6 fecal specimen was converted to $20 \%$ (wt/vol) suspensions in phosphate-buffered saline (PBS) (0.01 M, pH 7.4) and clarified by centrifugation at $10,000 \mathrm{~g}$ for $10 \mathrm{~min}$. The supernatant was then subjected to purification by passage through microfilters with a pore size of $0.22 \mu \mathrm{m}$ (Millipore, Japan) to remove possible bacteria and parasites. A $1.5 \mathrm{ml}$ aliquot of supernatant was used to infect five 15-day 


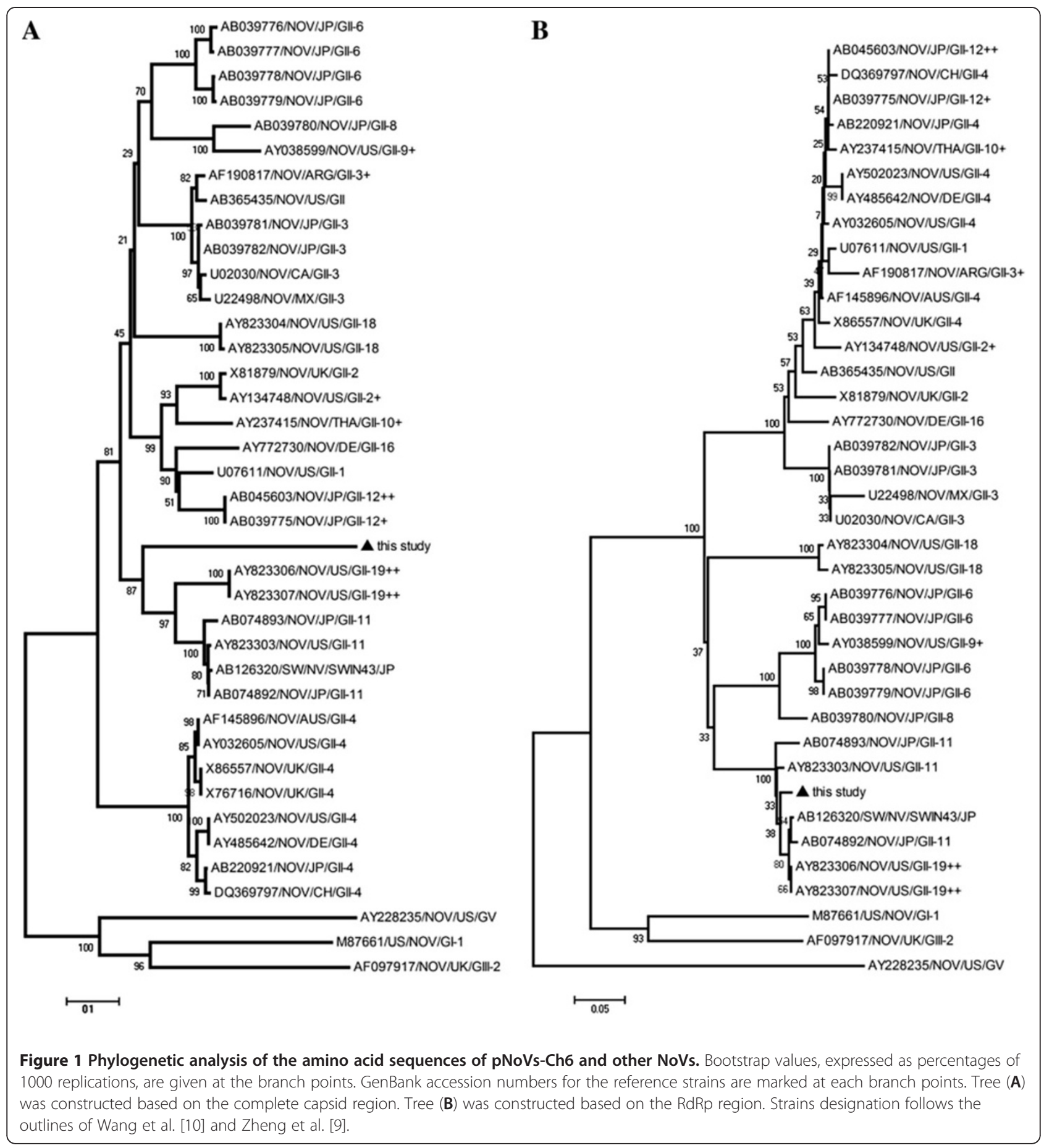

old piglets through oral inoculation. Another three piglets were only inoculated with PBS as controls. The piglets were investigated every 4 hours during the first day, and fecal samples were collected from each piglet every day. For the experimental group, one piglet was euthanized at post-inoculation day (PID) 1, 2, 4, 6, 10; meanwhile, one was euthanized at PID 1, 4, 10 for the control group. The intestinal contents were collected for RNA testing using RT-PCR, and intestinal segments were excised and immediately immersed in the different fixatives for histological examination. Tissues for histological examination were fixed in $10 \%$ neutral buffered formalin, routinely processed, sectioned at a thickness of $7 \mu \mathrm{m}$, and stained with hematoxylin and eosin. The tissue sections were examined and compared with negative controls. 


\section{Results}

Result of PoNoV detection

Result of detection indicated that two of the fecal samples were positive for PoNoV, one of which was coinfected with porcine circovirus. The others were negative for PoNoV, porcine circovirus type 2, porcine rotavirus, porcine transmissible gastroenteritis virus, porcine sapovirus, and porcine epidemic diarrhea virus. The fecal sample that was positive for only PoNoV was selected for further genome amplification and experimental infection.

\section{Genome organization and recombination analysis}

The entire genome of this PoNoV strain (named pNoVsCh6, GenBank accession number: HQ392821) consisted of 7531 nucleotides, excluding the poly (A) tail. Similar to previously reported NoVs strains, pNoVs-Ch6 has three open reading frames (ORFs), encoding 1693, 547 and 253 amino acids (aa), respectively. It shared $88 \%$ nucleotide homology with AB126320 over the entire genome sequence. The highest nucleotide homology over the RdRp sequence was shared with AB126320 (89\%) and then with an American porcine strain AY823303 (88\%).

A phylogenetic tree based on the predicted amino acid sequence of the complete capsid region showed that pNoVsCh6 was separated from known porcine GII strains (GII-11, GII-18 and GII-19) forming a new branch by itself (Figure 1A), which suggested that this strain may represent a novel genotype in GII group. However, phylogenetic analysis based on the RdRp region gave a different grouping result, where pNoVs-Ch6 was grouped into the GII-11 cluster (Figure 1B). This finding suggested that this virus strain may simultaneously be a recombinant. To confirm the finding and detect the breakpoint where the recombination event occurred, we performed recombination analysis based on 3/end RdRp and the capsid sequence of pNoVs-Ch6 as the query sequence, and AY823303/GII-11 and AY823306/GII19 as the background sequences using RPD software. Results indicated that the breakpoint located in the RdRpcapsid junction region, and the major parent was AY823303 (Figure 2). The minor parent was not found which may be due to the limited number of NoVs sequences available in GenBank, though AY823306 had a high similarity with the query strain over a short fragment (Figure 2).

\section{Experimental infection of miniature pigs with PoNoV positive fecal suspensions}

Our results showed that all of the five piglets in the experimental group indicated evident symptoms of mild to moderate diarrhea at 0.5-1 PID. The clinical signs persisted for 2 to 6 days, and no piglet died. All of the fecal samples and intestinal contents of the experimental group piglets were positive for NoVs RNA. Mild to moderate villous atrophy, mild to moderate and multifocal villous fusion were observed in the small intestine of experimental group pigs that were euthanatized from PID 6 to PID 10 (Figure 3). The three piglets in the control groups showed no clinical symptoms and tested negative for NoVs RNA.

\section{Discussion}

NoVs has been detected in a wide range of species, including humans, porcine, mice, cows and lions. Because PoNoV are genetically and antigenically related to human GII NoVs, there are public health concerns of potential cross-species transmission and animal reservoirs for NoVs related to human NoVs [10,17]. So far, PoNoV have been exclusively detected in fecal samples of adult pig without clinical signs $[18,19]$, though in experiments with gnotobiotic piglets mild diarrhea occurred [10]. In the current study, gastroenteritis in piglets caused by a PoNoV strain was reported. Experimental infection of miniature pigs with PoNoV positive fecal suspensions confirmed that this virus strain can cause gastroenteritis in pigs. To our knowledge, this study is the first identification of a PoNoV strain that cause piglets gastroenteritis under near natural conditions.

The genome of RNA viruses often undergoes recombination and segmentation [20].

Recombination is a driving force of viral evolution and has been reported for many single-stranded RNA viruses, including NoVs. It has been reported that the recombination of influenza virus leads to generation of a new strain and increases the biological fitness of the virus and its pathogenicity [1].

Previous reports have shown that, in the genome of NoVs, recombination mostly occurs at the junction point of ORF1 and ORF2 which is referred to as 'hot spot' [21-23]. Results of phylogenetic analysis and recombination identification showed that pNoVs-Ch6 was a recombinant strain, and the breakpoint located in the RdRp-capsid junction region like most other NoVs recombination events. To the best of our knowledge, this is the first report of recombinant PoNoV in China.

The detection of PoNoV that can cause gastroenteritis in piglets from commercial pig farms under natural conditions, on one hand, raises questions about the putative epidemiologic role of PoNoV, and, on the other hand, this kind of infectious strain also can be used to improve research such as virus replication, vaccine development, which has been impeded by the lack of a cell culture system for the propagation of human NoVs. Whether the recombination increased the virulence of PoNoV still needs further research.

\section{Conclusion}

In conclusion, the results in this study demonstrated that recombinant new genotype PoNoV exised in pig 


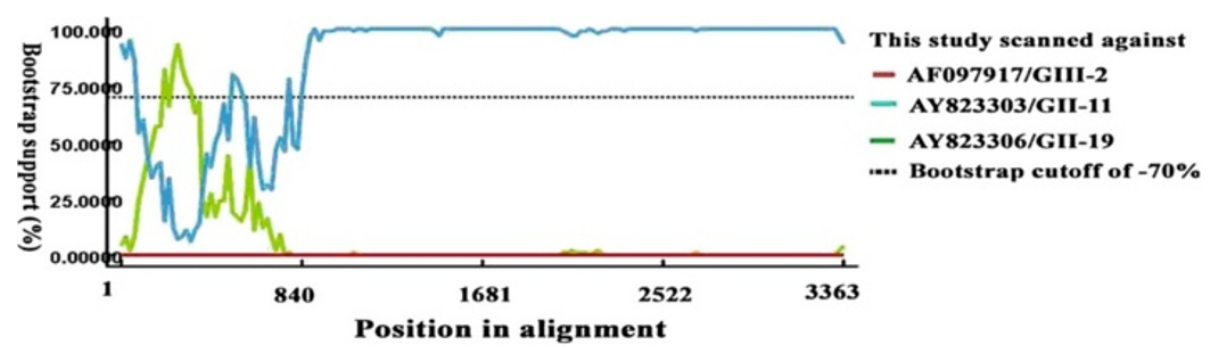

Figure 2 Identification of recombinant pNoVs-Ch6 strain. BOOTSCAN evidence for the recombination origin on the basis of pairwise distance, modeled with a window size 200, step size 25, and 100 Bootstrap replicates.
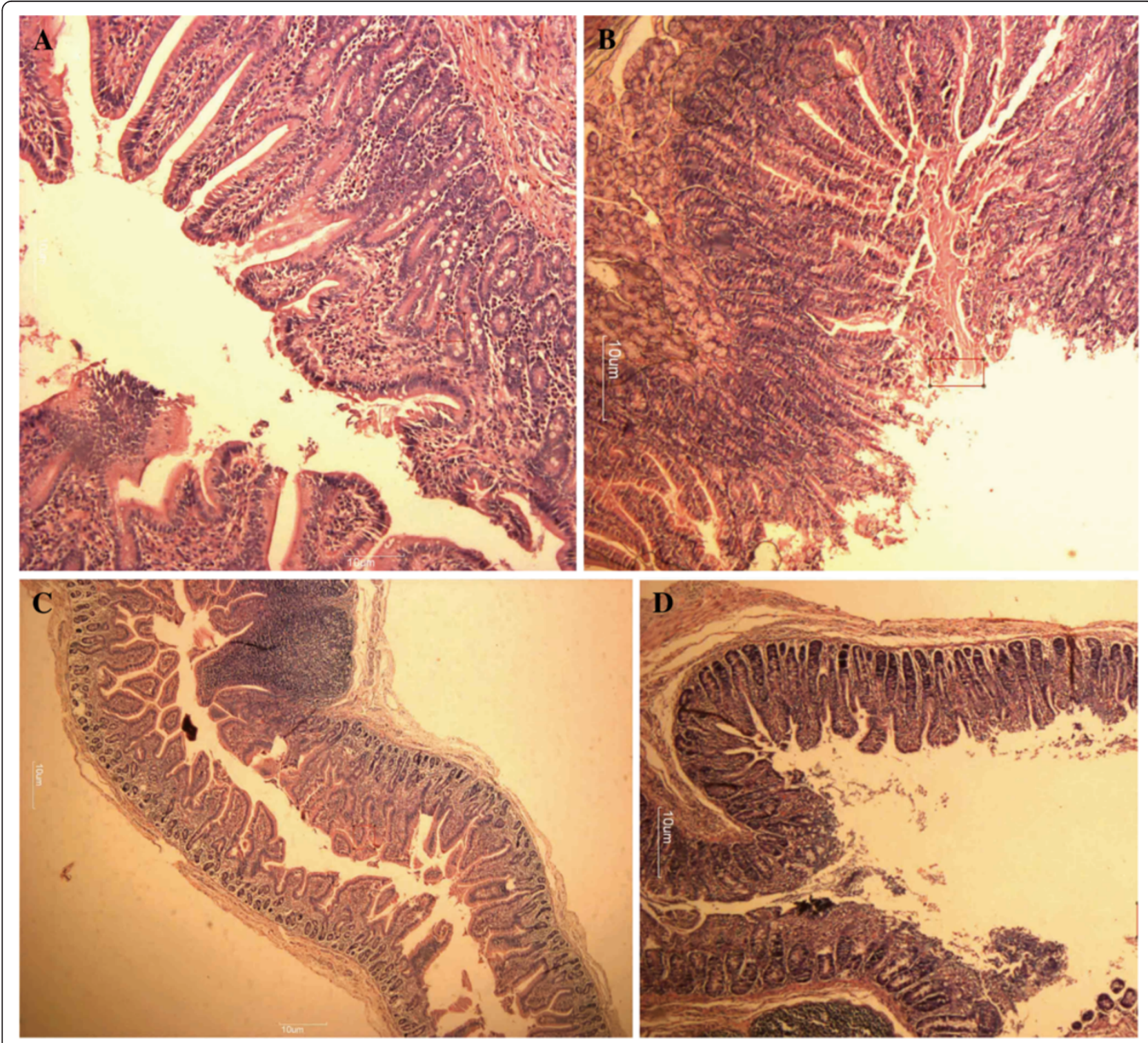

Figure 3 Histological lesions in the duodenum or jejunum of piglets following oral inoculation with NoVs or PBS. Hematoxylin and eosin stain. A, Normal-appearing, long villi of the duodenum from a PBS-inoculated pig; B, Villi of the duodenum showing mild villous atrophy from the NoVs-inoculated pig; C, Normal-appearing, long villi of the jejunum from a PBS-inoculated pig; D, Villi of the jejunum showing mild villous atrophy from the NoVs inoculated pig. Bar $=10 \mu \mathrm{m}$. 
herd of China, which cause diarrhea in pigs in nature condition. This find raised questions about the putative epidemiologic role of PoNoV.

\section{Authors' contributions}

Author Quan Shen, Wen Zhang, Shixing Yang, Yan Chen, Zhibiao Yang and Li Cui performed the experiments. Author Xiuguo Hua, Quan Shen and Wen Zhang designed the experiments. Quan Shen, and Wen Zhang performed the analysis and interpretation of data. Quan Shen wrote the manuscript. All the author approve submission of this manuscript to BMC Veterinary Research.

\section{Acknowledgments}

We thank Kelly Scheuer for assistance in linguistic revision of the manuscript. This work was supported by grants from the National Natural Science Foundation of China No. 31070132 and No.31270186, Key Project of Shanghai Science and Technology Committee of China under Grant No.10JC1406500, Natural Science Foundation of Jiangsu Province No. BK2012289, Research Fund for the Doctoral Program of Higher Education of China No. 20113227120008, and the Professional Research Foundation for Advanced Talents of Jiangsu University.

\section{Author details}

${ }^{1}$ School of Medical Science and Laboratory Medicine, Jiangsu University, 301 Xuefu Road, Zhenjiang, Jiangsu 212013, People's Republic of China. ${ }^{2}$ Key laboratory of Veterinary Biotechnology, School of Agriculture and Biology, Shanghai JiaoTong University, 800 Dongchuan Road, Shanghai, People's Republic of China. ${ }^{3}$ Food Animal Health Research Program, Ohio Agricultural Research and Development Center, The Ohio State University, Wooster, $\mathrm{OH}$ 44691-40961, USA.

Received: 6 January 2011 Accepted: 22 August 2012

Published: 3 September 2012

\section{References}

1. Fields BN, Knipe DM, Howley PM: Fields virology. 5th edition. Philadelphia; London: Wolters Kluwer Health / Lippincott Williams \& Wikins; 2007.

2. Liu BL, Lambden PR, Gunther H, Otto P, Elschner M, Clarke IN: Molecular characterization of a bovine enteric calicivirus: relationship to the Norwalk-like viruses. J Virol 1999, 73(1):819-825.

3. Jiang $X$, Wang $M$, Wang $K$, Estes MK: Sequence and genomic organization of Norwalk virus. Virology 1993, 195(1):51-61.

4. Sosnovtsev SV, Belliot G, Chang KO, Prikhodko VG, Thackray LB, Wobus CE, Karst SM, Virgin HW, Green KY: Cleavage map and proteolytic processing of the murine norovirus nonstructural polyprotein in infected cells. J Virol 2006, 80(16):7816-7831.

5. Belliot G, Sosnovtsev SV, Mitra T, Hammer C, Garfield M, Green KY: In vitro proteolytic processing of the MD145 norovirus ORF1 nonstructural polyprotein yields stable precursors and products similar to those detected in calicivirus-infected cells. J Virol 2003, 77(20):10957-10974.

6. Mattison K, Shukla A, Cook A, Pollari F, Friendship R, Kelton D, Bidawid S, Farber JM: Human noroviruses in swine and cattle. Emerg Infect Dis 2007, 13(8):1184-1188

7. Martella V, Campolo M, Lorusso E, Cavicchio P, Camero M, Bellacicco AL, Decaro N, Elia G, Greco G, Corrente M, et al: Norovirus in captive lion cub (Panthera leo). Emerg Infect Dis 2007, 13(7):1071-1073.

8. Martella V, Lorusso E, Decaro N, Elia G, Radogna A, D'Abramo M, Desario C, Cavalli A, Corrente M, Camero M, et al: Detection and molecular characterization of a canine norovirus. Emerg Infect Dis 2008, 14(8):1306-1308

9. Zheng DP, Ando T, Fankhauser RL, Beard RS, Glass RI, Monroe SS: Norovirus classification and proposed strain nomenclature. Virology 2006, 346(2):312-323.

10. Wang QH, Han MG, Cheetham S, Souza M, Funk JA, Saif L: Porcine noroviruses related to human noroviruses. Emerg Infect Dis 2005, 11(12):1874-1881.

11. Farkas T, Nakajima S, Sugieda M, Deng X, Zhong W, Jiang X: Seroprevalence of noroviruses in swine. J Clin Microbiol 2005, 43(2):657-661.
12. Jiang $X$, Huang PW, Zhong WM, Farkas T, Cubitt DW, Matson DO: Design and evaluation of a primer pair that detects both Norwalk- and sapporo-like caliciviruses by RT-PCR. J Virol Methods 1999, 83(1-2):145-154.

13. Jeong C, Park SI, Park SH, Kim HH, Park SJ, Jeong JH, Choy HE, Saif LJ, Kim SK, Kang Ml, et al: Genetic diversity of porcine sapoviruses. Vet Microbiol 2007, 122(3-4):246-257.

14. Yang JS, Song DS, Kim SY, Lyoo KS, Park BK: Detection of porcine circovirus type 2 in feces of pigs with or without enteric disease by polymerase chain reaction. J Vet Diagn Invest 2003, 15(4):369-373.

15. Kumar S, Tamura K, Nei M: MEGA3: integrated software for molecular evolutionary genetics analysis and sequence alignment. Brief Bioinform 2004, 5(2):150-163

16. Martin D, Rybicki E: RDP: detection of recombination amongst aligned sequences. Bioinformatics 2000, 16(6):562-563.

17. Guo M, Chang KO, Hardy ME, Zhang Q, Parwani AV, Saif L: Molecular characterization of a porcine enteric calicivirus genetically related to Sapporo-like human caliciviruses. J Virol 1999, 73(11):9625-9631.

18. van Der Poel WH, Vinje J, van Der Heide R, Herrera MI, Vivo A, Koopmans MP: Norwalk-like calicivirus genes in farm animals. Emerg Infect Dis 2000, 6(1):36-41.

19. Scipioni A, Mauroy A, Vinje J, Thiry E: Animal noroviruses. Vet J 2008, 178(1):32-45.

20. Domingo $E$, Holland JJ: RNA virus mutations and fitness for survival. Annu Rev Microbiol 1997, 51:151-178.

21. Ambert-Balay K, Bon F, Le Guyader F, Pothier P, Kohli E: Characterization of new recombinant noroviruses. J Clin Microbiol 2005, 43(10):5179-5186.

22. Bull RA, Hansman GS, Clancy LE, Tanaka MM, Rawlinson WD, White PA: Norovirus recombination in ORF1/ORF2 overlap. Emerg Infect Dis 2005, 11(7):1079-1085.

23. Jiang X, Espul C, Zhong WM, Cuello H, Matson DO: Characterization of a novel human calicivirus that may be a naturally occurring recombinant. Arch Virol 1999, 144(12):2377-2387.

\section{doi:10.1186/1746-6148-8-155}

Cite this article as: Shen et al:: Recombinant porcine norovirus identified from piglet with diarrhea. BMC Veterinary Research 2012 8:155.

\section{Submit your next manuscript to BioMed Central and take full advantage of:}

- Convenient online submission

- Thorough peer review

- No space constraints or color figure charges

- Immediate publication on acceptance

- Inclusion in PubMed, CAS, Scopus and Google Scholar

- Research which is freely available for redistribution 